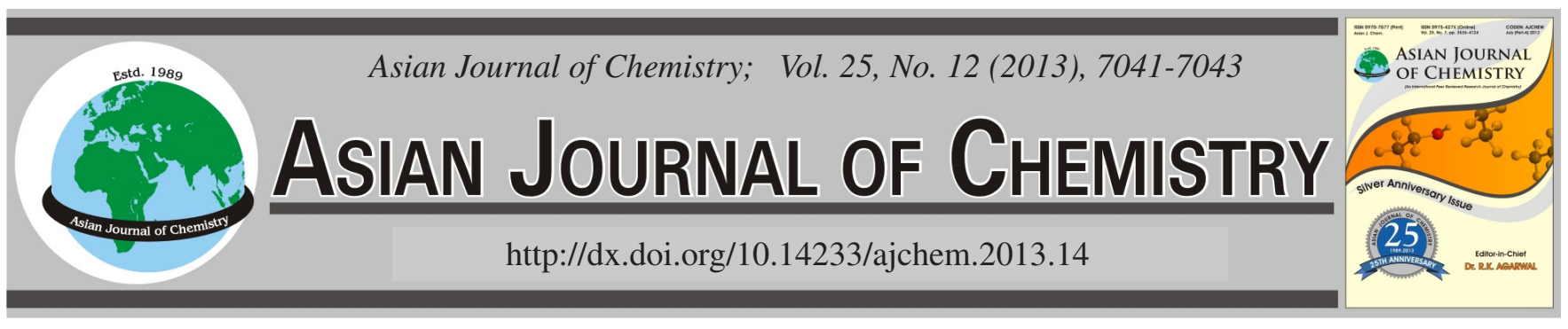

\title{
Methodology to Specify Software Requirements in Assessment System for Decommission of Nuclear Facilities $\dagger$
}

Hae Sang Song ${ }^{1}$, Kwan-Seong Jeong ${ }^{2}$, Dongunn Hyun ${ }^{2}$, Geun-Ho Kim², Byeong-Seon Choi ${ }^{2, *}$ and Jeikwon Moon ${ }^{2}$

${ }^{1}$ Computer Engineering Department, Seowon University, 377-3 Musimseoro, Heungdeok-gu, Cheongju, Chungbuk 361-742, Reublic of Korea ${ }^{2}$ Decontamination and Decommissioning Research Division, Korea Atomic Energy Research Institute, 989-111 Daedeok-daero, Yuseong-gu, Daejeon 305-353, Republic of Korea

*Corresponding author: Fax: +82 42 8682975; E-mail: bschoi@kaeri.re.kr

AJC-13636

\begin{abstract}
This paper deals with a methodology to specify software requirements as well as the top level requirements of the decommissioning assessment system of nuclear facilities. It then surveys related technologies required to build the system. Specifically the two major parts of the system include the assessment technology for radiation exposure to both the workers and the dismantling equipment and the process simulation of physical dismantling jobs such as moving, cutting and transporting. Also important is analyzing the 3 Dimentional interference between physical objects in the course of the jobs. Remote handling equipment to reduce the radiation exposure of workers is also considered. A trade-off study of many alternative processes can be supported using the system under consideration. This paper is expected to contribute to the development of requirements of the decommissioning process assessment system.
\end{abstract}

Key Words: Decommissioning assessment system, Nuclear radiation, Requirement specification, Simulation.

\section{INTRODUCTION}

It is well known that a requirement analysis is the most important part of system development, especially for systems that cost a lot of money and time ${ }^{1}$. It becomes more critical when the system under consideration is conceptually new and requires edge-technologies. The decommissioning assessment system of nuclear facilities is one of such systems that have high risks during development.

A number of nuclear facilities are expected to be decommissioned in several decades around the world ${ }^{2}$. As dismantling of nuclear facilities such as nuclear power plants is a major challenge faced with the clean-up of former nuclear sites, we need to increase the efficiency, reduce costs and optimize planning. To meet these needs, we have to verify the scenario key points, take into account unexpected situations and provide technical solutions. A simulation is a good means of visualizing and therefore understanding the constraints in testing different alternatives and is a way to train workers prior to intervention. As the lifetime of several Korean nuclear power plants will come to an end in a decade, KAERI (Korea Atomic Energy Research Institute) is planning a project to build such an assessment system that can be used to provide innovative simulation tools for the dismantling process of the nuclear facilities.
Although there have been a few assessment systems ${ }^{3-6}$, it was surveyed that no tool satisfies all the needs of KAERI. With this in mind, the project might fall in to high risk without employing a systematic approach to elicit and specify the requirements of such a system, which is called requirement engineering.

This paper proposes and applies the requirement engineering process and specification methods for the decommissioning assessment system, which results in a set of key requirements as well as a conceptual architecture. We also analyze the technological feasibility required to build the system. A project roadmap is also proposed to reduce developmental risks.

\section{ENGINEERING METHODOLOGY}

Requirement engineering process: We adopt partially NASA's systems engineering process to elicit and define our requirements. The process starts with defining the scope of the system; we first identify the need, objectives, mission, operational scenario and stake-holders. Then we define toplevel system requirements and architecture of the system. By iterative requirement analysis with the architecture decomposition we refine the system requirements to those of subsystem requirement specification. Whenever we decompose

$†$ Presented to the 4th International Symposium on Applications of Chemical and Analytical Technologies in Nuclear Industries, Daejeon, Korea 


\begin{tabular}{|c|c|c|}
\hline \multicolumn{3}{|c|}{$\begin{array}{c}\text { TABLE-1 } \\
\text { FUNCTIONAL REQUIREMENT DECOMPOSITION }\end{array}$} \\
\hline SR Level 1 & SR Level 2 & Feasible technologies \\
\hline \multirow{8}{*}{ SR1. 3D Simulation } & 1.1 Nuclear facility model & 3D modeling/digital mock-up [6] \\
\hline & 1.2 Worker model & 3D modeling/behaviour/HCI \\
\hline & 1.3 Equipment model & 3D modeling/behaviour/MMI \\
\hline & 1.4 3D simulation core & 3D simulation engine $[7] /$ physical engine \\
\hline & 1.5 Interactive operation & $\mathrm{HCI} /$ simulate 3D models \\
\hline & 1.6 Process capture/replay & Event $\log$ / replay of a process \\
\hline & 1.7 Destruction model & Cut, disassemble, transport / change models \\
\hline & 1.8 Dose visualization & Dose assessment / 3D visualization \\
\hline \multirow{7}{*}{ SR2. Assessment } & 2.1 Process management & Process edit / WBS for each workers \\
\hline & 2.2 Process assessment & Assess processes for does, cost, time, safety \\
\hline & 2.3 Work breakdown & Database, extraction for cost/work support \\
\hline & 2.4 Waste assessment & Amount of waste, waste cost \\
\hline & 2.5 Safety assessment & Physical damage \\
\hline & 2.6 Cost assessment & Cost of worker, equipment, time \\
\hline & 2.7 Dose assessment & CEA dose formula [8] \\
\hline \multirow{3}{*}{ SR3. Training (+visualization) } & 3.1 Worker virtual reality & VR [7,9] / avatar on 3D engine /Haptics \\
\hline & 3.2 Equipment virtual reality & MMI - manipulator / 3D engine/Haptics \\
\hline & 3.3 Destruction reality & 3D engine / physical engine \\
\hline \multirow{3}{*}{ SR4. On-site support (+training) } & 3.4 Worker support & HUD / placement/job/report \\
\hline & 3.5 Equipment handling & Remote manipulation/camera/job/report \\
\hline & 3.5 On-site visualization & Virtual - live synchronization/3D engine \\
\hline
\end{tabular}

the system architecture, requirements of the upper level components are flow down, allocated, or derived from the technological limitation to the low level requirements of each sub-system. It should be accompanied by interface requirements between components. In this way we can finally get a requirement specification document of each subsystem with the related interface requirements, which a detailed engineer uses for the design of a component. Technology, budget and time feasibility should be analyzed before the system requirement analysis.

Requirement analysis and specification methods: User requirements come from various sources such as scope document, stake-holder interests, regulations, related business documents, knowledge of the domain experts, benchmarks of similar systems, or purely artificial sources. Once eliciting the user requirements from these sources, we analyze them to specify the system requirements that are referenced by engineers or designers. We used various analysis methods of specifying the system requirements: The requirement decomposition, functional analysis, component decomposition, N-square chart, database modeling, control flow and functional flow ${ }^{1}$. Traceability is most important between the outcomes. We applied these methods iteratively to specify the assessment system requirements.

Requirement analysis and specification result: By applying the requirement engineering methodology above, we can determine the scope of the project, top level functional requirements and the conceptual architecture as follows.

Need statement: First, we specify the required statement as follows: "The Korean government needs to prepare the decommissioning of old nuclear power plants in a high radioactive environment in the near future. As dismantling is a very risky and costly process, an assessment system is necessary to assess the various dismantling scenarios to obtain the optimal one, to train workers before the actual execution and to support on-site workers to minimize human risks and reduce the cost and time".
Therefore the goal of the project is to obtain a decommissioning process assessment system, which can be extended to training and on-site work support.

Objective of the assessment system: The objectives of the system to meet the goal include the following three:

(1) Assessment of processes in terms of time, cost, waste, dose and safety of workers.

(2) Training of workers prior to actual destruction.

(3) Support of dismantling works including remote equipment handling.

Operational scenarios: Brief operation scenarios are identified as follows:

Assessment: Either an interactive or scenario-based dismantling process is simulated in a $3 \mathrm{D}$ simulation engine, which is coincidently accompanied by an assessment of dose exposure to workers, the estimation of waste generated during the work, the cost of the process, the physical safety of workers doing their jobs and the time necessary to finish the whole process.

Training: Workers are exercising their job of an optimal process using the MMI (man-machine interface) of the tools or equipment in an immersive virtual reality environment.

On-site support: Each worker on site is told their job description through a HUD (head-up display or something like) and reports their job progress to the system. The worker controlling remote handling equipment sees the on-site situation including the job description on the screen and reports the job progress.

Top-level functional requirement analysis and technological feasibility study: Table-1 identifies the first two levels of functional requirements of the assessment system with their technological feasibilities. The functional requirements should not specify the technologies to implement them, which restrains the engineer's opportunities for the design choices.

The system requirement consists of four top-level functions: 3D simulation, assessment, training and on-site support, each 
of which is decomposed into its level 2 functional requirements. Feasible technologies in the third column are identified to check if the requirements are implementable with existing technologies. The top three key technologies are categorized into a 3D game, virtual reality and augmented reality. The technologies for an assessment functionality are very domain specific. As a result, we conclude that all requirements are feasible using current technologies according to the survey of the current edge-technologies. However, the actual cost of implementation depends on the quality of the team involved in the development.

Architecture definition: Fig. 1 shows the conceptual architecture of the system to meet the functional requirements. The core of the system is a 3Dimensional simulation engine that consists of the visualization of 3D models and a physical engine that simulates the real-world physics for 3D objects. The dose simulation module calculates the worker or equipment radiation exposure level during their job in real-time or batch mode. The worker/equipment movement is simulated by the $3 \mathrm{D}$ engine, which is controlled by a predefined scenario or interactively. Multiple users control the workers and equipment interactively, which is recorded in the scenario database for a batch mode assessment. The destruction model simulates the cut, disassembly, packing of waste and transport. An object divided into pieces by equipment such as water jet is reflected into a 3D model database and waste database. Based on this core architecture, virtual reality technology and augmented reality technology enable the training and on-site support. The interfaces between modules are analyzed by an N-square chart, which is specified into an interface requirement document.

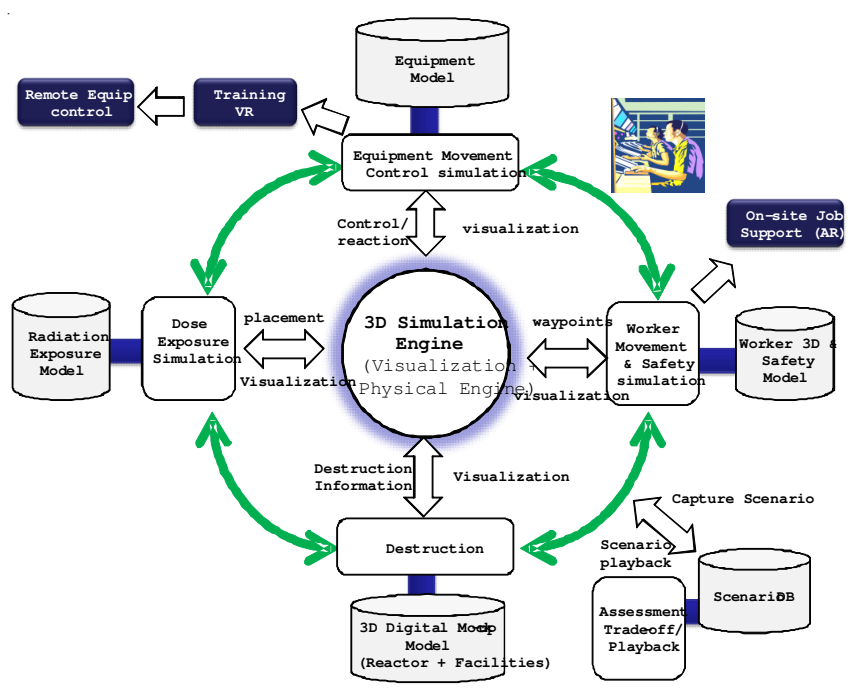

Fig. 1. Conceptual architecture of the assessment system
Actually, the core technology of the assessment system is a simulation of three types: 1) a constructive simulation that is purely synthetic, 2) a virtual simulation that involves a human interaction in the constructive simulation loop, 3) and a live simulation that collaborates with a real-world environment.

\section{Conclusion}

This paper described the methodology to specify the requirements of a decommissioning assessment system of nuclear facilities as well as an abstracted description of the results. First of all the most important thing is to define the scope of the system. Along with this, four top level functional requirements with their feasible technologies were identified. The top level conceptual architecture helps to refine the requirements into detailed subsystem requirements and interface requirements among decomposed subsystems. This specification methodology proposed is expected to contribute to specify successfully the requirements of the decommissioning process assessment system.

\section{REFERENCES}

1. National Aeronautics and Space Administration. NASA Systems Engineering Handbook (2007).

2. J. Samseth, A. Banford, B. Batandjieva-Metcalf, M.C. Cantone, P. Lietava, H. Peimani and A. Szilagyi, Closing and Decommissioning Nuclear Power Reactors-Another Look Following the Fukushima Accident, UNEP Year Book, pp. 35-49 (2012).

3. Y. Iguchi, Y. Kanehira, M. Tachibana and T. Johnsen, J. Nuclear Sci. Technol., 41, 367 (2004).

4. J.B. Thevenon, NARVEOS - A New Tool Supporting ALARA Studies. A Presentation on IAEA Training Course on Decommissioning Dose Assessment and Dose Optimization, MARCOULE CEA Center (2011).

5. F. Vermeersch, Radiat. Protection Dosimetry, 115, 294 (2005).

6. S.-K. Kim, H.-S. Park, K.-W. Lee and C.-H. Jung, Ann. Nucl. Energy, 33, 1227 (2006).

7. Z. Liu, M. Peng and Q. Zhao, J. Comp., 5, 343 (2011).

8. K.V. Subbaiah and R. Sarangapani, Ann. Nucl. Energy, 35, 2234 (2008).

9. C. Chabal, J. Mante and J. Idasiak, Int. J. Adv. Intelligent System, 4, 343 (2011). 